\title{
Study on the Perishable Product's Pricing Decision With Overconfident Consumers in the Dual-Channel Setting
}

\author{
Ying $\mathrm{Li}^{1} \&$ Guihang Guo ${ }^{1}$ \\ ${ }^{1}$ School of English for International Business, Guangdong University of Foreign Studies, Guangzhou, China \\ Correspondence: Guihang Guo, School of English for International Business, Guangdong University of Foreign \\ Studies, No.2 Baiyundadaobei, Guangzhou 510420, China. Tel: 86-139-2242-3319.
}

Received: June 16, 2020

Accepted: August 5, 2020

Online Published: August 24, 2020

doi:10.5430/ijba.v11n5p20

URL: https://doi.org/10.5430/ijba.v11n5p20

\begin{abstract}
With the development of internet, the online shopping mode has become more popular among consumers, and the online direct selling becomes more common. Besides buying products from traditional stores, consumers could get the product directly from the manufacturer online. In the dual channel setting, the competition becomes fiercer. Retailer should focus more on the price decision and take suitable pricing strategy to increase its profit. In this paper, consumer's overconfidence behavior is incorporated into perishable products' pricing decision in the partially integrated dual channel setting. Through the analysis of consumer's decision making process, this paper constructs the model for partially integrated manufacturer and retailer under the mean and precision overconfidence scenarios, conducts the optimal analysis, and analyzes the effect of consumer's overconfidence level on the optimal wholesale, retail and direct selling prices. We conclude that, no matter consumers are mean-overconfident or precision-overconfident; there are optimal wholesale price, direct sale price and retail price. Business enterprises should enhance their information collection capability and adopt some marketing measures to influence consumer's overconfidence level in order to increase the sales revenue.
\end{abstract}

Keywords: pricing, overconfident consumers, dual-channel

\section{Introduction}

With the development of Internet, the online shopping mode has become more popular among consumers, and the online direct selling has become more common as well. Besides buying products from traditional stores, consumers could get products directly from manufacturers online. For example, consumers could pay for the room at the hotel online. They could also make reservations on the websites of hotels or travel agencies. As for the airline ticket reservation, there are also two channels.

In the dual channel setting, the competition becomes fiercer. Retailers should focus more on the price decisions and take suitable pricing strategies to increase their profit. Consumers are overconfident in their purchase decisions. For example, they will have biased belief in their estimation. In consideration of overconfidence, what will be the retailer's optimal retail price and manufacturer's optimal wholesale and direct sale price?

As for the previous studies, they are mainly about the pricing decision of manufacturers and retailers in the direct sale and dual channel. Chiang (2003) studied the pricing problem in the dual-channel setting with consumer utility theory. Dumrongsiri (2008) studied members' pricing and ordering decision in the dual-channel supply chain. Yao and Liu (2004) analyzed the Bertrand price competition model and Stackelberg competition model. Chen Yun (2008) studied retailer's pricing behavior with two-stage gaming model in the dual-channel setting. At the first stage, retailers set the product price in the traditional and e-commerce channel, aiming to maximize the profit. At the second stage, consumers decided whether and where to buy based on their valuations and prices. Zhong et al (2012) present a model of an overconfident newsvendor who has both biased belief on mean and variance of demand. Wang et al (2015) investigated the impact of the retailer's overconfident behavior on supply chain performance. Chen et al (2016) investigated how the manufacturer made the pricing and production decision simultaneously and provided incentives to the sales agent so the agent worked hard to sell the product. They found that, as the level of overconfidence increased, the sales agent's efforts, the manufacturer's pricing and production decisions, as well as the expected profit deviate far away from the rational scenario. 
The difference between our research and these previous researches is that, we study the retailer's pricing decision in consideration of consumer overconfidence. Here, we assume that the retailers sell the perishable products in the dual-channel setting. The partially integrated dual channel is chosen for analysis. In this structure, besides selling the products to the retailer in the traditional channel, the manufacturer uses online selling channel as well. In consideration of consumer overconfidence, what will be the perishable products' pricing decision of the partially integrated manufacturer and retailer? What will be the effect of overconfidence on the perishable products' retail price, wholesale price and direct sale price? Based on the setting of the overconfident consumers in Li et al (2016), this study analyzes consumer's decision making process under the mean and precision overconfidence scenarios. By using the backward deduction, we analyze the retailer's retail price, partially integrated manufacturer's wholesale price and direct sale price, and the effects of consumer's overconfidence level on these optimal prices are given. Wu \& Hu (2018) incorporated retailers' overconfident behaviors into supply chain network dual channel equilibrium model which included supply markets, distribution markets and retail markets and analyzed the impacts of favorable and unfavorable market information on the order decision of overconfident retailers.

\section{Model Setting}

There is a dual-channel supply chain with one manufacturer, one retailer and consumers. Consumers could be overconfident, or regular (without overconfidence). The selling channel structure is shown in Figure 1. Suppose the selling period is single, the manufacturer and retailer both sell the perishable product in this period. Retailer's selling price in the traditional retail channel is $p_{r}$, and the direct sale price of the manufacturer in the direct sale channel is $p_{d}$. The wholesale price and cost of the product is $w$ and $c$ respectively. For the retailer and manufacturer, the handling cost of the leftover at the end of the selling period and the penalty cost of the stock-out are not considered.

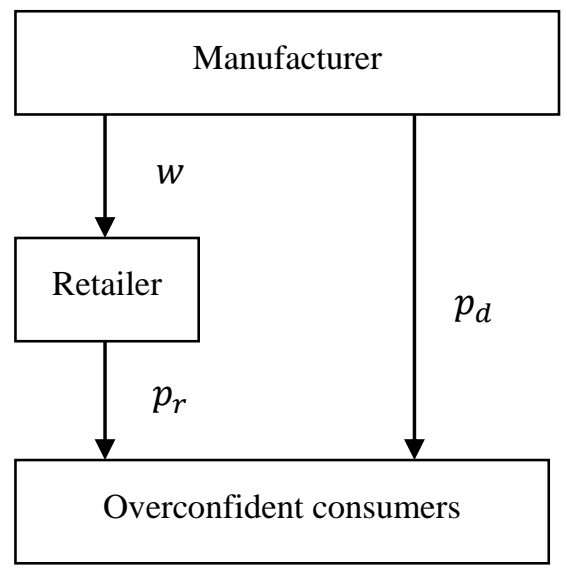

Figure 1. The partially integrated dual-channel selling structure

There are two different forms of overconfidence, which are mean overconfidence and precision overconfidence (Croson et al, 2008). Products sold online are not touchable before the delivery, so the valuation for them in the online channel is different from that in the traditional retail channel. In the dual channel setting, consumer overconfidence is shown not only in the product valuation, but also in the acceptable level of the channel. Now we analyze the consumer purchase process under the mean and precision overconfidence, and construct the profit models.

\subsection{Profit Model Under Mean Overconfidence Scenario}

Regular consumer's valuation on the product in the retailer channel is set as $V$, with the mean $\mu_{v}$, variance $\mu_{v}$. We set $V_{O M}$ as the mean-overconfident consumer's valuation on the product in the traditional retail channel. The overconfidence level is $\rho$, where $0 \leq \rho \leq 1$. Based on overconfidence definition in Croson et al (2008), we have the formulation:

$$
V_{O M}=V+\rho
$$

Regular consumer's valuation of the product in the traditional retail channel is set as $V$. According to Liang and Huang (1998), his valuation of the product in direct sale channel is $\eta V$, where $\eta$ is consumer's acceptance level of 
the direct sale channel. Here, $0<\eta<1$. Overconfident consumer will overestimate his acceptance level of the channel. Mean-overconfident consumer's acceptance level of the direct sale channel is set as $\eta_{O M}$. Here $\eta_{O M}=\eta+$ $\rho$.

We assume that the consumer is risk neutral and has at most one unit demand of the product. Regular consumer's valuation of the product follows the uniform distribution on $[0,1]$. The market capacity is standardized to be 1 . By simple calculation, we could get that mean-overconfident consumer's valuation of the product follows the uniform distribution on $[\rho, 1+\rho]$, with the probability density function $k_{O}(v)=1$.

In the selling period, mean-overconfident consumers compare utilities on two channels, and then they will decide whether to buy and where to buy. For the overconfident consumers, the utility from buying in the traditional retail channel is $V_{O M}-p_{r m}$, the utility from buying in the direct sale channel is $\eta_{O M} V_{O M}-p_{d m}$. In the case of $V_{O M}-$ $p_{r m} \geq \eta_{O M} V_{O M}-p_{d m}$, consumers are more willing to buy in the traditional retail channel. In the selling period, the number of mean-overconfident consumers who buy from the direct sale channel is:

$$
Q_{D M}=\int_{\frac{p_{d m}}{\eta_{O M}}}^{\frac{p_{r m}-p_{d m}}{1-\eta_{O M}}} k_{O}(v) d v=\frac{p_{r m}-p_{d m}}{1-\eta_{O M}}-\frac{p_{d m}}{\eta_{O M}}
$$

The number of mean-overconfident consumers who buy from the traditional retail channel is:

$$
Q_{R M}=\int_{\frac{p_{r m}-p_{d m}}{1-\eta_{O M}}}^{1+\rho} k_{O}(v) d v=1+\rho-\frac{p_{r m}-p_{d m}}{1-\eta_{O M}}
$$

So, the expected profit of the retailer is:

$$
\Pi_{R M}=Q_{R M}\left(p_{r m}-w_{m}\right)
$$

The expected profit of the partially integrated manufacturer is:

$$
\Pi_{D M}=Q_{R M}\left(w_{m}-c\right)+Q_{D M}\left(p_{d m}-c\right)
$$

Here $p_{r m}, p_{d m}$ and $w_{m}$ refer to the perishable product's retail price, direct sale price and wholesale price respectively under mean overconfidence scenario.

\subsection{Profit Model Under Precision Overconfidence Scenario}

Similar to the analysis of mean-overconfident consumer's purchase process, $1-\gamma$ is set as the overconfidence level of precision-overconfident consumer, where $0<\gamma<1$. Precision-overconfident consumer's valuation of the product in the traditional retail channel is:

$$
V_{O V}=\gamma V+(1-\gamma) \mu_{v}
$$

The precision-overconfident consumer's acceptance level of the direct sale channel is:

$$
\eta_{o V}=\gamma \eta+(1-\gamma) \mu_{\eta}
$$

For the precision-overconfident consumer, the utility from buying in the traditional retail channel is $V_{O V}-p_{r v}$, the utility from buying in the direct sale channel is $\eta_{O V} V_{O V}-p_{d v}$. In the case of $V_{O V}-p_{r v} \geq \eta_{O V} V_{O V}-p_{d v}$, consumers are more willing to buy in the traditional retail channel. The number of precision-overconfident consumers who buy from direct sale channel is:

$$
Q_{D V}=\int_{\frac{p_{d v}}{\eta_{O V}}}^{\frac{p_{r v}-p_{d v}}{1-\eta_{O V}}} g_{O}(v) d v=\frac{1}{\gamma}\left(\frac{p_{r v}-p_{d v}}{1-\eta_{O V}}-\frac{p_{d v}}{\eta_{O V}}\right)
$$

The number of precision-overconfident consumers who buy from the traditional retail channel is:

$$
Q_{R V}=\int_{\frac{p_{r v}-p_{d v}}{1-\eta_{O V}}}^{\frac{1+\gamma}{2}} g_{O}(v) d v=\frac{1}{\gamma}\left(\frac{1+\gamma}{2}-\frac{p_{r v}-p_{d v}}{1-\eta_{O V}}\right)
$$

So, the expected profit of the retailer is:

$$
\Pi_{R V}=Q_{R V}\left(p_{r v}-w_{v}\right)
$$


The expected profit of the partially integrated manufacturer is:

$$
\Pi_{D V}=Q_{R V}\left(w_{v}-c\right)+Q_{D V}\left(p_{d v}-c\right)
$$

Where $p_{r v}, p_{d v}$ and $w_{v}$ refer to the perishable product's retail price, direct sale price and wholesale price respectively under precision overconfidence scenario.

\section{The Optimal Decisions}

The partially integrated manufacturer and the retailer in the traditional retail channel get the optimal price decision through gaming. The gaming sequence is: the partially integrated manufacturer decides the wholesale price $w$ firstly, then the partially integrated manufacturer and retailer make their price decisions for each channel simultaneously. Now we use the backward deduction to analyze the optimal price decisions under two overconfidence scenarios.

\subsection{The Optimal Price Under Mean Overconfidence Scenario}

In this section, we analyze the optimal price decision of mean-overconfident consumers.

From the above analysis, we get:

$$
\begin{gathered}
\Pi_{R M}=\left(1+\rho-\frac{p_{r m}-p_{d m}}{1-\eta_{O M}}\right)\left(p_{r m}-w_{m}\right) \\
\Pi_{D M}=\left(1+\rho-\frac{p_{r m}-p_{d m}}{1-\eta_{O M}}\right)\left(w_{m}-c\right)+\left(\frac{p_{r m}-p_{d m}}{1-\eta_{O M}}-\frac{p_{d m}}{\eta_{O M}}\right)\left(p_{d m}-c\right)
\end{gathered}
$$

The first derivative of $\Pi_{R M}$ with respect to $p_{r m}$, and $\Pi_{D M}$ to $p_{d m}$ are both set as 0 , and then we have:

$$
\begin{aligned}
& \frac{\partial \Pi_{R M}}{\partial p_{r m}}=\frac{2 p_{r m}-w_{m}-1+\eta_{O M}-\rho+\rho \eta_{O M}-p_{d m}}{\eta_{O M}-1}=0 \\
& \frac{\partial \Pi_{D M}}{\partial p_{d m}}=\frac{-\eta_{O M} w_{m}+\eta_{O M} c+2 p_{d m}-c-\eta_{O M} p_{r m}}{\eta_{O M}\left(\eta_{O M}-1\right)}=0
\end{aligned}
$$

By solving the above equations, we get:

$$
\begin{gathered}
p_{r m}=\frac{-2 w_{m}-w_{m} \eta-w_{m} \rho-2+2 \eta+2 \rho \eta+2 \rho^{2}+c \eta+c \rho-c}{-4+\eta+\rho} \\
p_{d m}=\frac{-3 w_{m} \eta-3 w_{m} \rho+2 c \eta+2 c \rho-2 c+\rho \eta-\eta-\rho+\eta^{2}+\rho \eta^{2}+2 \rho^{2} \eta+\rho^{3}}{-4+\eta+\rho}
\end{gathered}
$$

By substituting (1) and (2) into $\Pi_{D M}$, taking the first derivative of $\Pi_{\mathrm{DM}}$ with respect to $w_{m}$ and setting it as 0 :

$$
\frac{\partial \Pi_{D M}}{\partial w_{m}}=\frac{\rho^{3}+2 \rho^{2} \eta+\rho^{2}-2 w_{m} \rho+2 \rho \eta+8 \rho+\rho \eta^{2}+c \rho+c \eta-16 w_{m}-2 w_{m} \eta+8 c+\eta^{2}+8}{(-4+\eta+\rho)^{2}}=0
$$

Then we get:

$$
w_{m}=\frac{\rho^{3}+2 \rho^{2} \eta+\rho^{2}+2 \rho \eta+8 \rho+\rho \eta^{2}+c \rho+c \eta+8 c+\eta^{2}+8}{2(\rho+\eta+8)}
$$

\subsection{The Optimal Price Decision in Precision Overconfidence Scenario}

In this section, we analyze the optimal price decision of precision-overconfident consumers.

From the above analysis, we get:

$$
\begin{gathered}
\Pi_{R V}=\frac{1}{\gamma}\left(\frac{1+\gamma}{2}-\frac{p_{r v}-p_{d v}}{1-\eta_{O V}}\right)\left(p_{r v}-w_{v}\right) \\
\Pi_{D V}=\frac{1}{\gamma}\left(\frac{1+\gamma}{2}-\frac{p_{r v}-p_{d v}}{1-\eta_{O V}}\right)\left(w_{v}-c\right)+\frac{1}{\gamma}\left(\frac{p_{r v}-p_{d v}}{1-\eta_{O V}}-\frac{p_{d v}}{\eta_{O V}}\right)\left(p_{d v}-c\right)
\end{gathered}
$$

The first derivative of $\Pi_{R V}$ with respect to $p_{r v}, \Pi_{D V}$ to $p_{d v}$ are both set as 0 , and then we have:

$$
\frac{\partial \Pi_{R V}}{\partial p_{r v}}=\frac{4 p_{r v}-2 w_{v}-1+\eta_{O V}-\gamma+\gamma \eta_{O V}-2 p_{d v}}{2 \gamma\left(\eta_{O V}-1\right)}=0
$$




$$
\frac{\partial \Pi_{D V}}{\partial p_{d v}}=\frac{-\eta_{O V} w_{v}+\eta_{O V} c+2 p_{d v}-c-\eta_{O V} p_{r v}}{\gamma \eta_{O V}\left(\eta_{O V}-1\right)}=0
$$

By solving the above equations, we get:

$$
\begin{gathered}
p_{r v}=\frac{-1-c \mu_{\eta} \gamma+w_{v} \mu_{\eta} \gamma-c-w_{v} \gamma \eta-\gamma-2 w_{v}}{-4+\gamma \eta+\mu_{\eta}-\mu_{\eta} \gamma}+\frac{\mu_{\eta}+\gamma \eta+c \gamma \eta-w_{v} \mu_{\eta}+c \mu_{\eta}+\gamma^{2 \eta}-\mu_{\eta} \gamma^{2}}{-4+\gamma \eta+\mu_{\eta}-\mu_{\eta} \gamma} \\
p_{d v}=\frac{-6 w_{v} \gamma \eta-6 w_{v} \mu_{\eta}+6 w_{v} \mu_{\eta} \gamma+4 c \gamma \eta+4 c \mu_{\eta}-4 c \mu_{\eta} \gamma-4 c-\gamma^{2} \eta-\gamma \eta}{2\left(-4+\gamma \eta+\mu_{\eta}-\mu_{\eta} \gamma\right)}+\frac{\gamma^{2} \eta^{2}+2 \gamma \eta \mu_{\eta}+\gamma^{3} \eta^{2}-2 \gamma^{3} \eta \mu_{\eta}-\mu_{\eta}+\mu_{\eta}^{2}-\mu_{\eta}^{2} \gamma^{2}+\mu_{\eta} \gamma^{2}-\mu_{\eta}^{2} \gamma+\mu_{\eta}^{2} \gamma^{3}}{2\left(-4+\gamma \eta+\mu_{\eta}-\mu_{\eta} \gamma\right)}
\end{gathered}
$$

By substituting (3) and (4) into $\Pi_{D V}$, taking the first derivative of $\Pi_{D V}$ with respect to $w_{v}$ and setting it as 0 :

$$
\begin{aligned}
& \frac{\partial \Pi_{D V}}{\partial w_{v}}=\frac{-2 \gamma^{3} \eta \mu_{\eta}+\mu_{\eta}^{2} \gamma^{3}+\gamma^{3} \eta^{2}+\gamma^{2} \eta^{2}-\mu_{\eta}^{2} \gamma^{2}+2 \gamma \eta \mu_{\eta}-4 w_{v} \gamma \eta+2 c \gamma \eta}{2 \gamma\left(-4+\gamma \eta+\mu_{\eta}-\mu_{\eta} \gamma\right)^{2}} \\
&+\frac{4 w_{v} \mu_{\eta} \gamma-2 c \mu_{\eta} \gamma-\mu_{\eta}^{2} \gamma+8 \gamma+16 c-32 w_{v}+2 c \mu_{\eta}+8+\mu_{\eta}^{2}-4 w_{v} \mu_{\eta}}{2 \gamma\left(-4+\gamma \eta+\mu_{\eta}-\mu_{\eta} \gamma\right)^{2}}=0
\end{aligned}
$$

Then we get:

$$
\begin{aligned}
w_{v}=\frac{-2 \gamma^{3} \eta \mu_{\eta}+}{+} \mu_{\eta}^{2} \gamma^{3}+\gamma^{3} \eta^{2}+\gamma^{2} \eta^{2}-\mu_{\eta}^{2} \gamma^{2}+2 \gamma \eta \mu_{\eta} \\
4\left(\gamma \eta-\mu_{\eta} \gamma+8+\mu_{\eta}\right) \\
+\frac{2 c \gamma \eta-2 c \mu_{\eta} \gamma-\mu_{\eta}^{2} \gamma+8 \gamma+16 c+2 c \mu_{\eta}+8+\mu_{\eta}^{2}}{4\left(\gamma \eta-\mu_{\eta} \gamma+8+\mu_{\eta}\right)}
\end{aligned}
$$

Summarizing the above analysis, we get the following results.

(1) When consumers are mean-overconfident:

The wholesale price of the perishable product for the partially integrated manufacturer is:

$$
\frac{\rho^{3}+2 \rho^{2} \eta+\rho^{2}+2 \rho \eta+8 \rho+\rho \eta^{2}+c \rho+c \eta+8 c+\eta^{2}+8}{2(\rho+\eta+8)}
$$

The direct sale price is:

$$
\frac{-3 w_{m} \eta-3 w_{m} \rho+2 c \eta+2 c \rho-2 c+\rho \eta-\eta-\rho+\eta^{2}+\rho \eta^{2}+2 \rho^{2} \eta+\rho^{3}}{-4+\eta+\rho}
$$

The retailer's retail price is:

$$
\frac{-2 w_{m}-w_{m} \eta-w_{m} \rho-2+2 \eta+2 \rho \eta+2 \rho^{2}+c \eta+c \rho-c}{-4+\eta+\rho}
$$

Where $w_{m}$ is the perishable product's wholesale price under the mean overconfidence scenario.

(2) When consumers are precision-overconfident:

The wholesale price of the perishable product for the partially integrated manufacturer is:

$$
\frac{-2 \gamma^{3} \eta \mu_{\eta}+\mu_{\eta}^{2} \gamma^{3}+\gamma^{3} \eta^{2}+\gamma^{2} \eta^{2}-\mu_{\eta}^{2} \gamma^{2}+2 \gamma \eta \mu_{\eta}}{4\left(\gamma \eta-\mu_{\eta} \gamma+8+\mu_{\eta}\right)}+\frac{2 c \gamma \eta-2 c \mu_{\eta} \gamma-\mu_{\eta}^{2} \gamma+8 \gamma+16 c+2 c \mu_{\eta}+8+\mu_{\eta}^{2}}{4\left(\gamma \eta-\mu_{\eta} \gamma+8+\mu_{\eta}\right)}
$$

The direct sale price of the perishable product is:

$$
\begin{array}{r}
\frac{-6 w_{v} \gamma \eta-6 w_{v} \mu_{\eta}+6 w_{v} \mu_{\eta} \gamma+4 c \gamma \eta+4 c \mu_{\eta}-4 c \mu_{\eta} \gamma-4 c-\gamma^{2} \eta-\gamma \eta}{2\left(-4+\gamma \eta+\mu_{\eta}-\mu_{\eta} \gamma\right)} \\
+\frac{\gamma^{2} \eta^{2}+2 \gamma \eta \mu_{\eta}+\gamma^{3} \eta^{2}-2 \gamma^{3} \eta \mu_{\eta}-\mu_{\eta}+\mu_{\eta}^{2}-\mu_{\eta}^{2} \gamma^{2}+\mu_{\eta} \gamma^{2}-\mu_{\eta}^{2} \gamma+\mu_{\eta}^{2} \gamma^{3}}{2\left(-4+\gamma \eta+\mu_{\eta}-\mu_{\eta} \gamma\right)}
\end{array}
$$

The retailer's retail price of the perishable product is:

$$
\frac{-1-c \mu_{\eta} \gamma+w_{\mathrm{v}} \mu_{\eta} \gamma-c-w_{\mathrm{v}} \gamma \eta-\gamma-2 w_{\mathrm{v}}+\mu_{\eta}+\gamma \eta+c \gamma \eta-w_{\mathrm{v}} \mu_{\eta}+c \mu_{\eta}+\gamma^{2 \eta}-\mu_{\eta} \gamma^{2}}{-4+\gamma \eta+\mu_{\eta}-\mu_{\eta} \gamma}
$$


Where $w_{\mathrm{v}}$ is the wholesale price of the perishable product under the precision overconfidence scenario?

\section{Numerical Examples}

In order to make the effect of consumer overconfidence level on these optimal prices clearer, numerical examples are used for illustration.

(1) Mean overconfidence consumer

According to the setting of $\eta$ in Liang and Huang (1998) and the relationship of the parameters, we set $\eta=0.8$, $\mathrm{c}=2$. In order to show the effect of consumer's mean overconfidence level on the pricing decisions of retailer and partially integrated manufacturer, the overconfidence level $\rho$ is increased from 0 to 1 with an increment of 0.1 . Figures $2 a), 2 b$ ) and 2c) show the effect of consumer's mean overconfidence level on the optimal wholesale price, the optimal direct sale price, and the optimal retail price respectively. From the figures, we could see that the optimal wholesale and direct sale price increase with consumer overconfidence level. For the partially integrated manufacturer, when the overconfidence level increases, manufacturer should increase its wholesale price and direct sale price. For the retailer, when the overconfidence level is lower than a threshold, retailer could increase its retail price when consumer overconfidence level increases; when the overconfidence level is higher than this threshold, retailer should decrease its retail price when consumer overconfidence level increases.

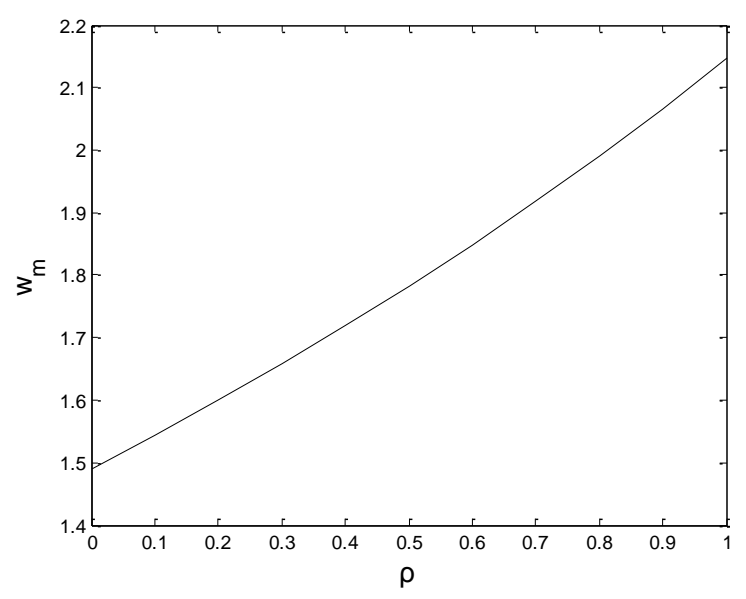

Figure 2a. The effect of consumer's mean overconfidence level on the optimal wholesale price

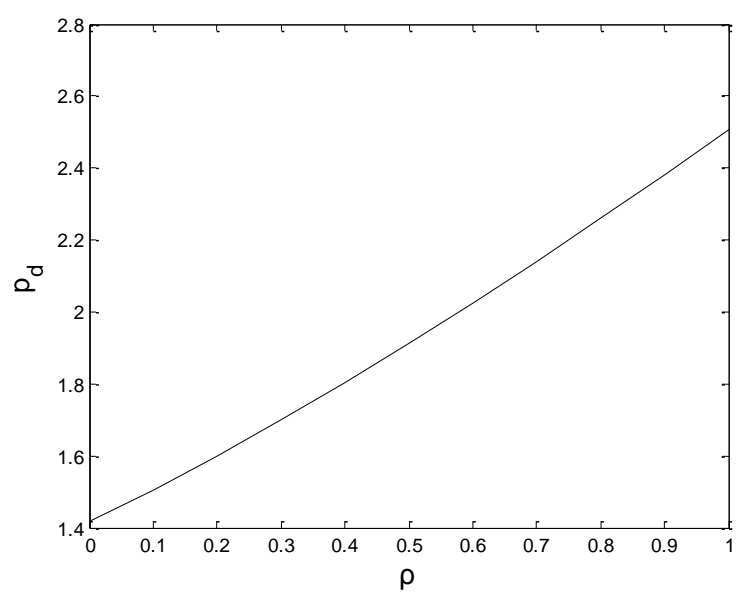

Figure 2b. The effect of consumer's mean overconfidence level on the optimal direct sale price 


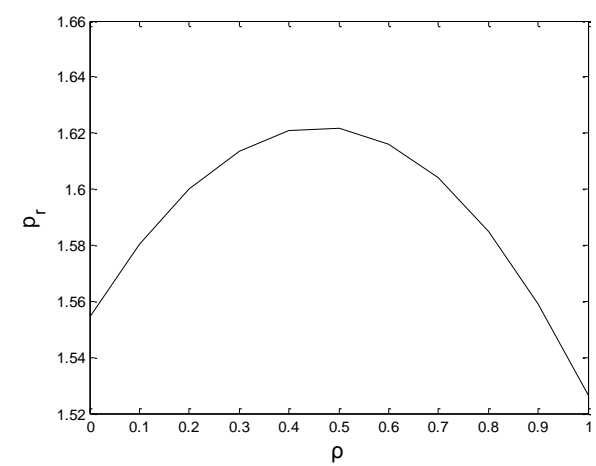

Figure 2c. The effect of consumer's mean overconfidence level on the optimal retail price

(2) Precision overconfidence consumer

According to the relationship of the parameters, we set $c=2$, and $\eta$ follows the uniform distribution on [0.5,1]. In order to see the effect of precision overconfidence level on the pricing decisions of retailer and partially integrated manufacturer, $\gamma$ is increased from 0 to 1 with an increment of 0.1. Figures 3a), 3b), and 3c) show the effect of consumer's precision overconfidence level on the optimal wholesale price, the optimal direct sale price, and the optimal retail price respectively. From the figures, we could see that all the prices decrease when consumer overconfidence level increases. For the partially integrated manufacturer and retailer, when the overconfidence level increases, manufacturer and retailer should decrease their prices. This is different from the findings when consumers are mean-overconfident. It is also shown that the optimal wholesale price is a bit higher than optimal direct sale price. In reality, it might cause the retailer's speculation behavior. Instead of "by wholesale" way, the retailer could buy products directly from the direct sale channel and then resell them.

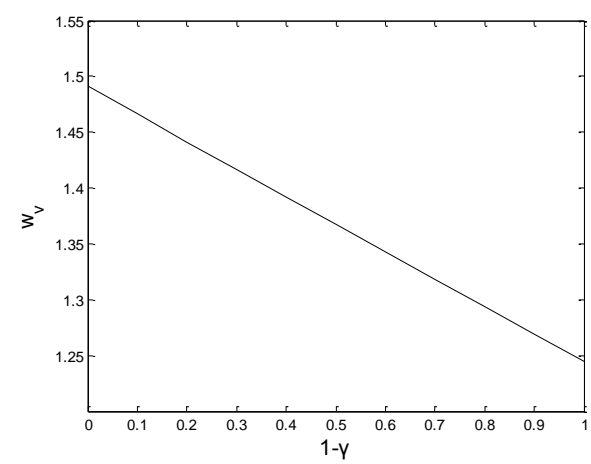

Figure 3a. The effect of consumer's precision overconfidence level on optimal wholesale price

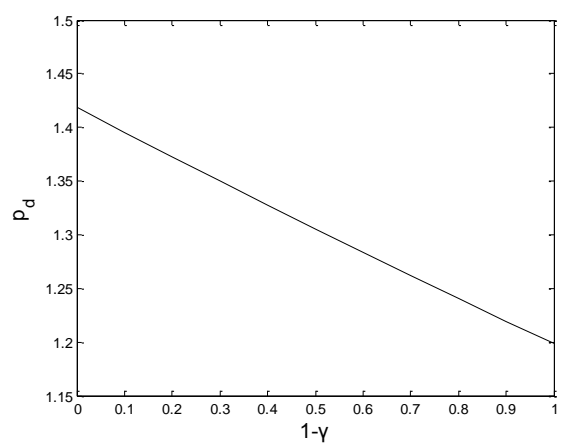

Figure 3b. The effect of consumer's precision overconfidence level on optimal direct sale price 


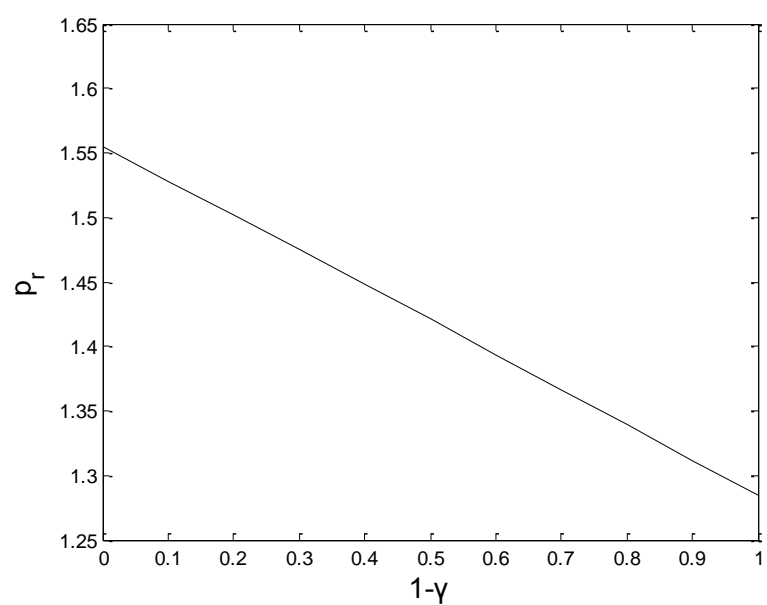

Figure 3c. The effect of consumer's precision overconfidence level on optimal retail price

\section{Concluding Remarks}

In the previous studies, Li et al (2016) analyzed the advance selling decisions with overconfident consumers, while Wu \& Hu (2018) analyzed the impacts of favorable and unfavorable market information on the order decision of overconfident retailers. In consideration of consumer's mean and precision overconfidence, this paper studies the pricing decision of retailer and partially integrated manufacturer in the partially integrated dual channel. Findings show that, no matter consumers are mean-overconfident or precision-overconfident, there are optimal wholesale price, direct sale price and retail price. Numerical examples are used to illustrate the effect of consumer overconfidence level on these optimal prices.

Dual-channel is very common in reality. Business enterprises should enhance the information collection capability. For example, they can collect consumers' historical data to classify consumers, and then enhance the competitive advantage. Furthermore, they could adopt some marketing measures to influence consumer's overconfidence level, aiming at increasing the revenue.

In the market, retailers know the consumers' demand and its trend better than others. With the improvement of retailer's capability, retailers could gain more negotiation power in the supply chain. Therefore, the retailer-leading game in the dual-channel setting could be considered for the further study. In reality, there are some other dual channel structures, like the vertically integrated dual channel, decentralized dual channel and horizontally integrated channel. The effect of consumer's overconfidence level on retailer and manufacturer's pricing decisions in these structures could be another extension for further studies.

\section{References}

Chen, K. G., Song, X. F., Wang, X. Y., \& Huang, M. (2016). Joint Pricing and Production Decisions with the Overconfident Sales Agent. Journal of Systems \& Management, 25(3), 468-476.

Chen, Y., Wang, H. Ch., \& Shen, H. Zh. (2008). Study on the pricing strategy of multi-channel retailer in internet environment. Journal of Industrial Engineering and Engineering Management, 1, 34-39.

Chiang, W. K., Chajed, D., \& Hess, J. D. (2003). Direct marketing, indirect profits: A strategic analysis of dual-channel supply-chain design. Management Science, 49(1), 1-20. https://doi.org/10.1287/mnsc.49.1.1.12749

Croson, D. C., Croson, R., \& Ren, Y. (2008). How to manage an overconfident newsvendor. Working Paper, UT Dallas.

Dumrongsiri, A., Fan, M., Jain, A., et al.. (2008). A supply chain model with direct and retail channels. European Journal of Operational Research, 187(3), 691-783. https://doi.org/10.1016/j.ejor.2006.05.044

Liang, T., \& Huang, J. (1998). An empirical study on consumer acceptance of products in electronic markets: a transaction cost model. Decision Support Systems, 24(1), 29-43. https://doi.org/10.1016/s0167-9236(98)00061-x

Wang, Z. G., Zhang, Zh. Ch., Li, Ch. F., Xu, L., et al.. (2015). Optimal ordering and disposing policies in the 
presence of an overconfident retailer: A Stackelberg game. Mathematical Problems in Engineering. https://doi.org/10.1155/2015/385289

Wu, Y. L., \& Hu, J. S. (2018). Supply Chain Network Dual Channel Equilibrium Model with Overconfident Retailers. Operations Research and Management Science, 27(1), 96-102. https://doi.org/10.12005/orms.2018.0015

Yao, D. Q., \& Liu, J. J. (2004). Competitive pricing of mixed retail and e-tail distribution channels. Omega-International Journal of Management Science, 33(3), 235-247. https://doi.org/10.1016/j.omega.2004.04.007

Ying, L., Shan, M. Y., \& Michael, Z. F. Li. (2016). Advance Selling Decisions with Overconfident Consumers. Journal of Industrial and Management Optimization, 12(3), 891-905. https://doi.org/10.3934/jimo.2016.12.891

Zhong, Y. W., Liu, Zh. R., Guo, J. S., \& Li, J. C. (2012). Research on Ordering Decision and Coordination of Overconfident Retailer Based on Newsvendor Model. Operations Research and Management Science, 21(3), 62-66. https://doi.org/10.3969/j.issn.1007-3221.2012.03.009

\section{Copyrights}

Copyright for this article is retained by the author(s), with first publication rights granted to the journal.

This is an open-access article distributed under the terms and conditions of the Creative Commons Attribution license (http://creativecommons.org/licenses/by/4.0/). 